\title{
Factors influencing the successful validation of transient phenomenon modelling
}

\author{
Ricardo Jauregui Telleria ${ }^{1}$, Ferran Silva ${ }^{1}$, Antonio Orlandi ${ }^{2}$, Hugh Sasse $^{3}$, Alistair Duffy ${ }^{3}$ \\ ${ }^{1}$ Universitat Politècnica de Catalunya, Spain ${ }^{2}$ University of L'Aquila, Italy, ${ }^{3}$ De Montfort University, UK \\ Corresponding author: apd@dmu.ac.uk
}

\begin{abstract}
An increased requirement for validation of computational electromagnetic simulation and modelling through the publication of IEEE Standard 1597.1 brings to light some interesting issues surrounding the validation of transients. The structure of a transient event has three particular regions of interest that can have an influence on the results, of which only two are generally well defined. These are the initial quiescent phase from $t=0$ to the transient event; the transient event itself up to the point where the energy has fallen to a predefined limit, and the post-transient phase where residual energy is still present in the system. This latter region is generally ill-defined and changes the way that a validation comparison should be made, from, for example a frequency domain coupling study where the region of interest is usually well defined. This study looks at the influence of the three regions on the validation results and suggests how the Feature Selective Validation (FSV) method can be applied in transient studies.
\end{abstract}

\section{INTRODUCTION}

In recent years electronic devices have increased and diversified, ranging from entertainment systems, control, security, safety, etc. Each of these systems usually has an associated a communications path, either by cable or by a radio link, most of which are digital. The increased density of systems and buses also increases the probability of mutual electromagnetic interferences.

For decades the analysis in the frequency domain has been useful enough for the analysis of many EMC phenomena, but the analysis of systems only in the frequency domain is not always enough to evaluate their immunity. One good example of the importance of analysis in the time domain is digital communications systems.

Digital communication systems are highly immune to continuous interferences thanks to the modern techniques of coding channel and complex protocols, but the digital system is weak in the face of a radiated transient as shown by numerous studies $[1,2,3]$. Due to this behaviour, the study of transient or impulsive noise is an interesting subject concerning EMC [4] as the characterisation of the 'victim' before, during and after the transient is an important in understanding the system.

While measurements can be an excellent way to study any system, there are inherent difficulties with such an approach: namely because of the cost and limitations of the equipment able to measure in the time domain with the required sensitivity and accuracy or due to being early in the design phase. For this reason, the numerical methods are a helpful addition to the design and analysis of digital systems. In recent years several studies have been published to obtain the distributions of electromagnetic fields in complex structures based on numerical methods $[5,6]$.

Having said that there are three regions to consider and two are well defined, it should also be noted that this definition is generally implicitly defined rather than explicitly defined, i.e. experienced engineers will have an intuitive understanding of the regions more frequently than these regions will be obtained from analysis. For example, consider figure 1, which shows a modelled and measured transient event. Some issues that have a direct impact on the comparison are:

- How long should the quiescent phase run (not labelled) prior to the transient event itself?

- At what point is it acceptable to say that the transient event has finished and the post-event, energy dissipation, phase has started?

- How long should the post-event phase run on for?

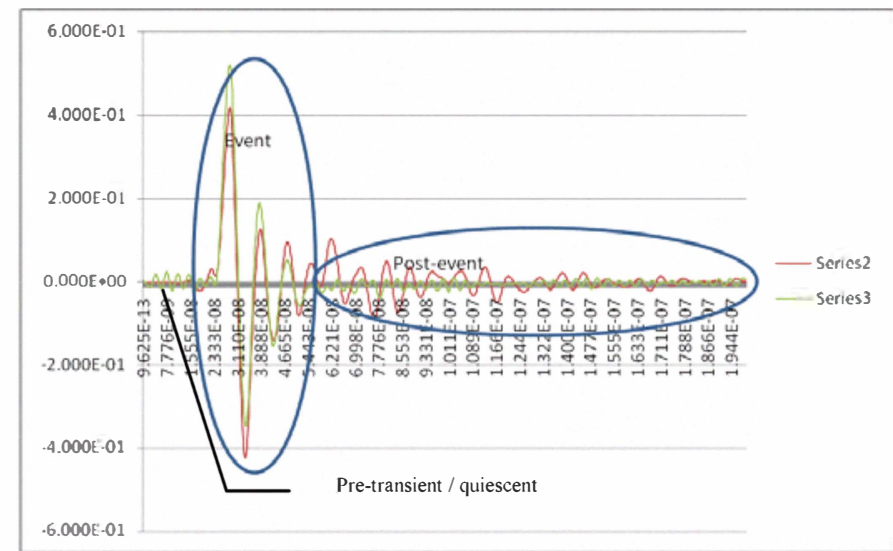

Fig. 1 Illustration of the regions of a transient event

This is usually a matter of personal preference for the engineers studying such phenomena. However, this is of great importance if the quality of the comparison between the model and measurements is to be determined quantitatively, which is required if the modelling approach used is to be validated according to IEEE standard 1597.1 [7]. Figure 1 of [7] shows that one route to validation of the simulations approach used to analyse transients in this way is to take the individual model, and compare this against measurements using the Feature Selective Validation (FSV) method.

FSV [8] is a heuristic that, by decomposing the data sets to be compared into trend and fine detail ('low Q' and 'high Q' features) comparisons and using distance metrics for this 
decomposed data and the derivatives of this data has been shown to produce a quantified comparison that is closely analogous to the comparison made by a group of experienced engineers [9].

In the rest of the paper, the next section will describe FSV in more detail and explore some of the issues of applying it to the transient phenomena; Section III will suggest an approach for formalising the comparison of transients and Section IV will draw this discussion to a conclusion.

\section{FSV AND TRANSIENT PHONEMENA}

FSV is defined, mathematically, in the following equations (reproduced from [7]). The overall 'goodness of fit' is called the GDM (Global Difference Measure) and this is made up of two components, the ADM (Amplitude Difference Measure) and the FDM (Feature Difference Measure). The original data for both data sets is filtered into a near-DC component (low pass filtered, labelled DC in the following equations) a lowfrequency component (band pass filtered, labelled Lo in the following equations) and a high-frequency component (high pass filtered, labelled $\mathrm{Hi}$ in the following equations)

$$
\operatorname{ADM}(n)=\left|\frac{\alpha}{\beta}\right|+\left|\frac{\chi}{\delta}\right| \exp \left\{\left|\frac{\chi}{\delta}\right|\right\}
$$

Where

$$
\begin{aligned}
& \alpha=\left(\left|L o_{1}(n)\right|-\left|L o_{2}(n)\right|\right) \\
& \beta=\frac{1}{N} \sum_{i=1}^{N}\left(\left(\left|L o_{1}(i)\right|+\left|L o_{2}(i)\right|\right)\right) \\
& \chi=\left(\left|D C_{1}(n)\right|-\left|D C_{2}(n)\right|\right) \\
& \delta=\frac{1}{N} \sum_{i=1}^{N}\left(\left(\left|D C_{1}(i)\right|+\left|D C_{2}(i)\right|\right)\right) \\
& F D M(f)=2\left(\left|F D M_{1}(f)+F D M_{2}(f)+F D M_{3}(f)\right|\right)
\end{aligned}
$$

Where

$$
\begin{aligned}
& F D M_{1}(f)=\frac{\left|L o_{1}^{\prime}(f)\right|-\left|L o_{2}{ }^{\prime}(f)\right|}{\frac{2}{N} \sum_{i=1}^{N}\left(\left(\left|L o_{1}{ }^{\prime}(i)\right|+\left|L o_{2}{ }^{\prime}(i)\right|\right)\right)} \\
& F D M_{2}(f)=\frac{\left|H i_{1}^{\prime}(f)\right|-\left|H i_{2}^{\prime}(f)\right|}{\frac{6}{N} \sum_{i=1}^{N}\left(\left(\left|H i_{1}{ }^{\prime}(i)\right|+\left|H i_{2}{ }^{\prime}(i)\right|\right)\right)} \\
& F D M_{3}(f)=\frac{\left|H i_{1}{ }^{\prime}(f)\right|-\left|H i_{2}{ }^{\prime \prime}(f)\right|}{\frac{7.2}{N} \sum_{i=1}^{N}\left(\left(\left|H i_{1}{ }^{\prime \prime}(i)\right|+\left|H i_{2}{ }^{\prime \prime}(i)\right|\right)\right)}
\end{aligned}
$$

Note that the primes and double primes indicate derivatives. Also, the use of $(n)$ and $(f)$ are to emphasize that the two sets of data are obtained differently, although the extent of the $\mathrm{ADM}$ and FDM is equal and equivalent to the original data to be compared.

$$
G D M(f)=\sqrt{A D M(f)^{2}+F D M(f)^{2}}
$$

A mean, single value, of ADM, FDM and GDM can be obtained by taking the means of equations $1-3$ respectively.

The resulting data can be categorised using natural language descriptors according to Table I

Table I FSV interpretation scale [7]

\begin{tabular}{|l|l|}
\hline FSV value (quantitative) & FSV interpretation (qualitative) \\
\hline Less than 0.1 & Excellent \\
\hline Between 0.1 and 0.2 & Very good \\
\hline Between 0.2 and 0.4 & Good \\
\hline Between 0.4 and 0.8 & Fair \\
\hline Between 0.8 and 1.6 & Poor \\
\hline Greater than 1.6 & Very poor \\
\hline
\end{tabular}

Table I allows the overall, single-value, quality measures resulting from FSV analysis to be described using familiar 'pegs'. However, it must be remembered that these are not absolute levels of acceptance or failure but simply descriptions to help in the communication of the quality of the comparisons.

The point-by-point data can be binned according to Table I creating a probability density function - a confidence histogram - which has been shown to provide a generally good level of agreement with a group assessment [9].

It will be noted in each of these equations that the denominator is a function of the weighted average intensity of the filtered data. The effect of this in transient events is that if the pre- and post-event regions are extended too far, they are close to zero in comparison to the main event. However, the main event is usually short lived, so the resultant is that excessive times devoted to the pre- and post-event regions reduce the denominator which effectively amplifies differences in the numerator resulting in FSV comparisons that are notably in less agreement than a visual inspection would be. The reason for this is that the visual inspection will ignore much of the pre- and post-event regions but FSV will process what it is given. Which means that the post-event region shown in Figure 2 has a considerable influence on the overall results.

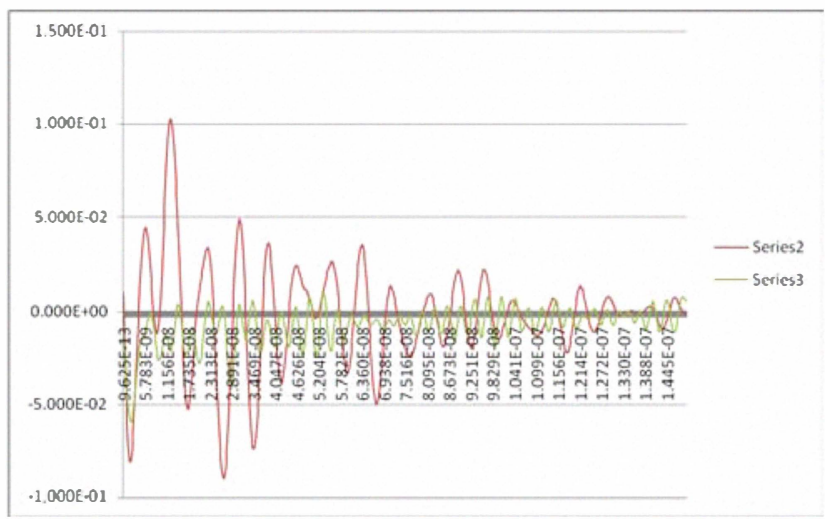

Fig. 2 Post-event region from Figure1 
The effect of the post transient region on the results can be seen from Figures 3 and 4 which show the ADM for the transient region and the post-transient region. The skewing of the results in the post-transient region can be clearly seen.

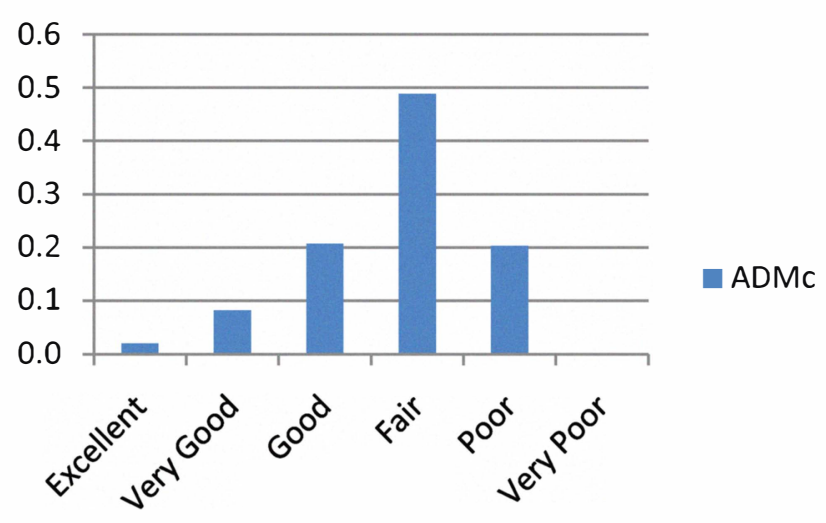

Fig. 3 ADM confidence histogram for the transient region

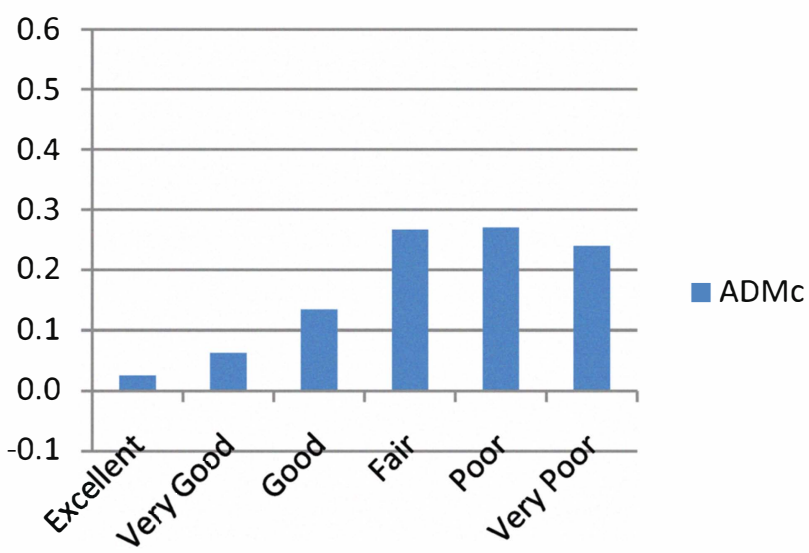

Fig. 4 ADM confidence histogram for the post-transient region.

While it would be tempting to ignore the post-transient region, this is not a sensible approach as the information contained in that data is helpful to indicate how the energy is stored and dissipated in the system once the source of that energy has been removed and, as such, is also valuable to the designer.

A second issue that may have an influence on the FSV result is the nature of the transient curves themselves: namely that there are a substantial number of zero crossings in the data. It will be noted from equations (1) and (2) that the differences used to construct the FSV metrics rely on differences of absolute values. As the data crosses zero, there will be an abrupt change of sign as the absolute value is taken. This is a factor of FSV that can be accounted for by preprocessing the data to be compared by offsetting the origin of the data so that it exists entirely in the positive or negative half planes. Providing the same offset is applied to both data sets (or all data sets if more than two are being cross compared) then there will be no effect on the veracity of the FSV results will.

The next section addresses both of these issues and proposes a method of weighted regions in order to

\section{A METHOD OF WEIGHTED REGIONS FOR TRANSIENT ANALYSIS}

The data shown in Figure 1 were compared using FSV. The GDM confidence histogram was obtained using the tool available from [10]. This is shown in Figure 5.

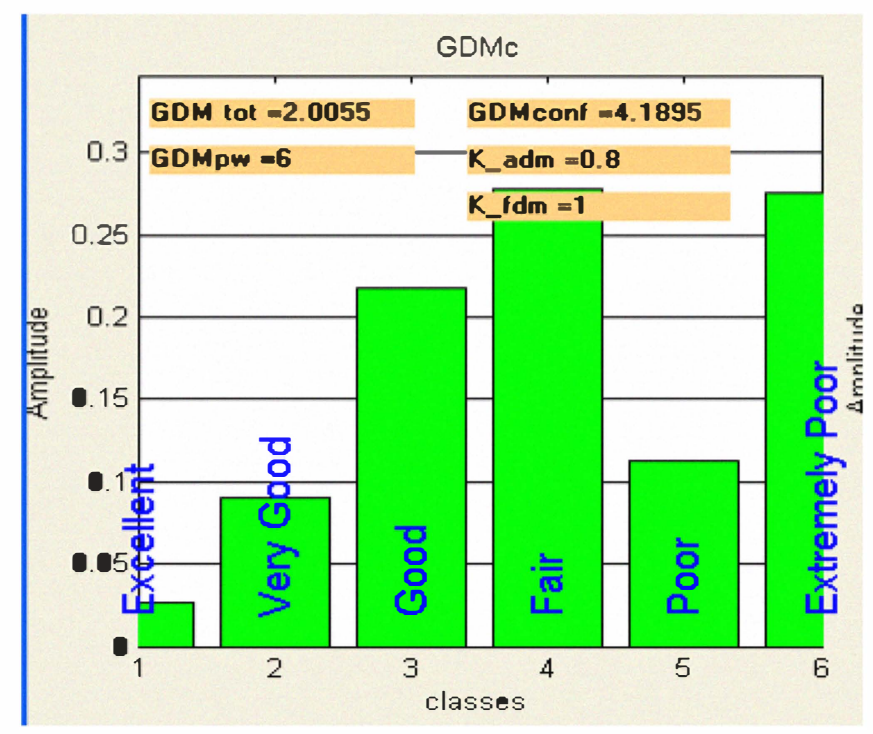

Fig. 5 GDM of original data.

The GDM clearly shows that there is a skewing towards 'Extremely (very) Poor'. It is anticipated that the cause of this is the large number of zero crossings in that data. A discussion of the results with other workers in the field agreed that a GDM of 2.0 (Very Poor) is an excessively harsh assessment of the comparison in Figure 1, even accounting for the long tail of relatively poorly agreeing post-transient phenomena. Figure 6 shows the effect of placing the data in the positive half plane using an offset equal to the maximum negative excursion of the data sets. The overall GDM, in this case, is 0.36 , i.e. 'Good', is more in agreement with the anticipated visual assessment.

For reasons of space, other results are not reproduced here, but it should also be noted that a relatively small offset of the data sets was sufficient to produce results very little different to those in Figure 6, similarly an offset of a magnitude greater made no difference to the FSV results compared with Figure 6. 


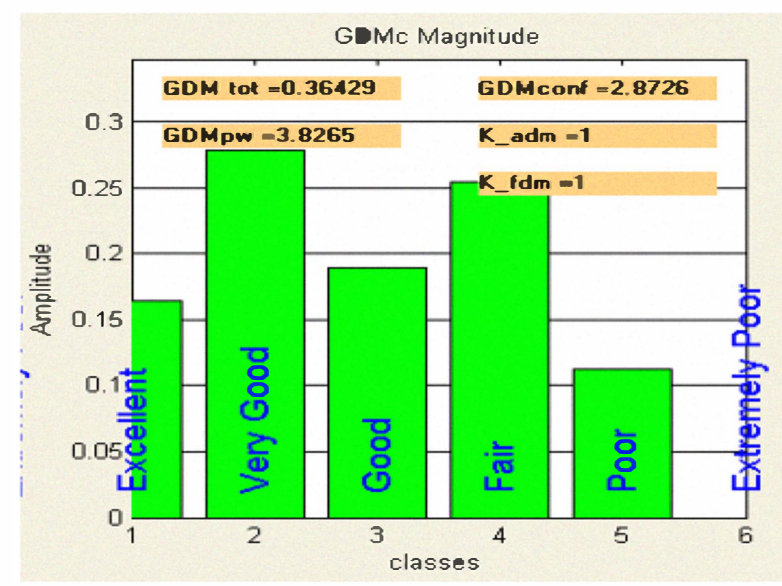

Fig. 6 GDM of original data placed entirely in the positive half plane

However, acknowledging that the three regions previously discussed do not have an equal weighting. However, as also discussed, these regions should not be ignored because they provide potentially useful information about the system being analysed. Hence, the three regions were separated according to the following rules.

- In order to obtain the length of the pre-transient region the maximum value of both signals is obtained, after accounting for any DC offset. The data is scanned back to a point where the amplitude is less than $5 \%$ of the peak (this is the Alternative Amplitude Point (AAP)) and the earliest of these is chosen for the boundary between the pre- transient and transient regions.

- The transient region is obtained by calculating the energy in the two data sets and extending the 'transient' window from the end of the pre-transient region to a point which contains $65 \%$ of the energy in the curves (the region of greatest extent is chosen if the two data sets do not coincide). It should be noted that the relatively low levels in the pre- and post-transient regions will have relatively little impact on this region.

- The post-transient region extends from the end of the transient region until the end of recorded time.

The FSV result was obtained by then taking the confidence histogram results from the three regions and combining them in the relative proportions of 5\% pre-transient, $70 \%$ transient and $25 \%$ post-transient. No account was taken of the relative number of data points in each region as the approach used here is not then highly dependent on the length of the pre- and post-transient regions. Although it must be noted that this may also have a skewing effect on the final data and is a topic for further investigation. The resulting GDM confidence histogram is given in Figure 7.

With no data offset, using the Weighted Region approach, the overall GDM was 0.77 ('Fair', borderline 'Poor') whereas, with both the offset and the Weighted Region approach, the overall GDM was reduced to 0.53 (a mid-rated 'Fair').

\section{CONCLUSION}

This paper has addressed the problems associated with comparing modelled and measured transient data with the aim of applying IEEE standard 1597.1. The principal conclusion from this work, summarised in this paper, is that the transient data should be offset to ensure it is in one half-plane and that a Weighted Region approach should be used to ensure that the comparison data from the three regions present in the transient data are captured but that this is done in a measured, objective, way, reducing the quality dependency on the lengths of the pre- and post-transient regions used.

The research undertaken for this paper prompts other avenues for investigation. These include undertaking a more formal analysis of the offset requirements and verifying, and modifying if necessary, the sizes and weightings of the Weighted Regions.

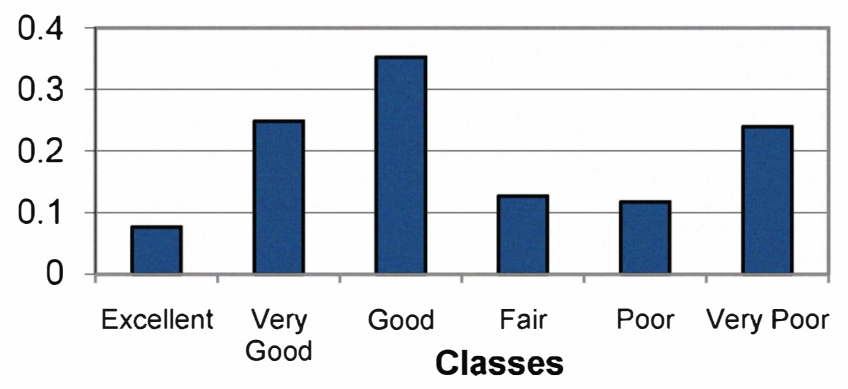

Fig. 7 Weighted Region GDM

\section{REFERENCES}

[1] S. Verdaguer, M. Fernández, A. Vidal and F. Silva. "EMI receiver plus digitizer RF transient measurements". EMC York 2004 International Conference and Exhibition July 2004, York, UK, pp 635-640.

[2] I. E. Portugues, P. J. Moore, I. A. Glover, and R. J.Watson. "Aportable wideband impulsive noise location system". 57(9):2059-2066, 2008 ISSN 0018-9456. doi: 10.1109/TIM.2008.917258

[3] Ana Vázquez Alejos(3) Iñigo Cuiñas (1), Manuel García Sánchez(2) "Wideband impulsive noise measurements in dvb-t and umts radio channels". IEEE,2002.

[4] Anzaldi, G., Quilez, M.; Riu, P. J. \& Silva, F. "Analysis of the Human Body Influence on a Bluetooth Link Inside a Vehicle". COST 286 Hamburg, 2004.

[5] R. Jauregui, F. Silva and P. Riu. "FDTD Analysis of the Distribution of a Transient Field inside a Car," EMC Europe Workshop 2007. June 2007, Paris, Francia.

[6] R. Jauregui, Marc Pous, Mireya Fernández, Pere J. Riu, Ferran Silva "Validation of low-level, high-frequency transient FDTD simulation with radiated field measurements". EMC Europe 2008. 6-09-2008. Hamburgo- Alemania.

[7] IEEE Std 1597.1 "Standard for the validation of computational electromagnetics simulation and modeling", February 2009, Piscataway, $\mathrm{NJ}$, USA.

[8] Duffy, A. P., Martin, A. J. M., Orlandi, A., Antonini, G., Benson, T. M and Woolfson, M. S., Feature Selective Validation (FSV) for validation of computational electromagnetics (CEM). Part I - The FSV method, IEEE Trans-EMC, Vol. 48 No. 3, Aug. 2006, pp. 449 - 459.

[9] A Orlandi, AP Duffy, B Archambeault, G Antonini, DE Coleby, S Connor "Feature Selective Validation (FSV) for validation of computational electromagnetics (CEM). Part II - Assessment of FSV performance" IEEE Trans-EMC Vol 48 No 3, Aug 2006 pp 460 - 467

[10] A Orlandi, "FSV 4.0.6",

http://uaqemc.ing.univaq.it/uaqeme/FSV_ID_4_0_6 